Research Article

\title{
Exact Solutions of the Space-Time Fractional Bidirectional Wave Equations Using the $\left(G^{\prime} / G\right)$-Expansion Method
}

\author{
Wei Li, Huizhang Yang, and Bin He \\ College of Mathematics of Honghe University, Mengzi, Yunnan 661100, China \\ Correspondence should be addressed to Wei Li; wellars@163.com
}

Received 15 March 2014; Accepted 9 June 2014; Published 23 June 2014

Academic Editor: Jin Liang

Copyright (c) 2014 Wei Li et al. This is an open access article distributed under the Creative Commons Attribution License, which permits unrestricted use, distribution, and reproduction in any medium, provided the original work is properly cited.

Based on Jumarie's modified Riemann-Liouville derivative, the fractional complex transformation is used to transform fractional differential equations to ordinary differential equations. Exact solutions including the hyperbolic functions, the trigonometric functions, and the rational functions for the space-time fractional bidirectional wave equations are obtained using the $\left(G^{\prime} / G\right)$ expansion method. The method provides a promising tool for solving nonlinear fractional differential equations.

\section{Introduction}

It has recently become more interesting to obtain exact solutions of fractional differential equations (FDEs). These equations have been proved to be an excellent tool in the modelling of many phenomena in various fields such as signal processing, viscoelastic flow, materials and mechanics, biology systems, anomalous diffusion, and medical [1-5]. Such methods as the variational iteration method, the expfunction method, the general Riccati equation, the fractional subequation method, and the first integral method have been proposed to solve the FDEs [6-13]. Here, it is worth to mention the $\left(G^{\prime} / G\right)$-expansion method $[14,15]$. The $\left(G^{\prime} / G\right)$ expansion method proposed by Wang et al. [16] is one of the most effective direct methods to obtain exact solutions of a large number of nonlinear evolution equations. Based on Jumarie's modified Riemann-Liouville derivative, the $\left(G^{\prime} / G\right)$ expansion method was further extended $[14,15,17-20]$ to find the solutions of fractional differential equations. In this paper, we will apply the $\left(G^{\prime} / G\right)$-expansion method to obtain more and new exact solutions for the space-time fractional bidirectional wave equations [21]:

$$
\begin{gathered}
D_{t}^{\alpha} v+D_{x}^{\alpha} u+u D_{x}^{\alpha} v+v D_{x}^{\alpha} u+a D_{x}^{\alpha} D_{x}^{\alpha} D_{x}^{\alpha} u \\
-b D_{x}^{\alpha} D_{x}^{\alpha} D_{t}^{\alpha} v=0, \quad 0<\alpha \leq 1, \\
D_{t}^{\alpha} u+D_{x}^{\alpha} v+u D_{x}^{\alpha} u+c D_{x}^{\alpha} D_{x}^{\alpha} D_{x}^{\alpha} v-d D_{x}^{\alpha} D_{x}^{\alpha} D_{t}^{\alpha} u=0,
\end{gathered}
$$

where $a, b, c$, and $d$ are real constants, $t$ is the elapsed time, $x$ represents the distance along the channel, the variable $u(x, t)$ is the dimensionless horizontal velocity, and $v(x, t)$ is the dimensionless deviation of the water surface from its undisturbed position. When $\alpha=1,(1)$ is the generalization of bidirectional wave equations, which was derived as a model equation describing the propagation of long waves on the surface of water with a small amplitude by Bona and Chen [22]. On the other hand, it is formally equivalent to the classical Boussinesq system and correct through first order with regard to a small parameter characterizing the typical amplitude to depth ratio [23]. Equation (1) for $\alpha=1$ is studied by many researchers; for instance, Chen [23] used the auxiliary ordinary equation method to obtain some exact solutions of (1) for $\alpha=1$ and the exact travelling wave solutions by 
Lee and Sakthivel [24] by using the modified tanh-coth function method. The exact solutions of the space-time fractional bidirectional wave equations (1) are only reported in [21]. Based on Jumarie's modified Riemann-Liouville derivative and the fractional Riccati equation $D_{\xi}^{\alpha} \phi(\xi)=\sigma+\phi^{2}(\xi)$, Lu [21] obtained the rational formal solutions of (1) by introducing a new general ansätz.

This paper is organized as follows. In Section 2, some basic properties of Jumarie's modified Riemann-Liouville derivative are given. In Section 3, the main steps of the $\left(G^{\prime} / G\right)$-expansion method are given. In Section 4 , we construct the exact solutions of (1) by the present method. Some conclusions are given in Section 5.

\section{Preliminaries}

In this section, we give some definitions and formulas of Jumarie's modified Riemann-Liouville derivative.

Jumarie $[25,26]$ defined the fractional derivative in the limit form

$$
f^{\alpha}(x)=\lim _{h \rightarrow 0} \frac{\Delta^{\alpha}[f(x)-f(0)]}{h^{\alpha}}, \quad 0<\alpha<1,
$$

where

$$
\Delta^{\alpha} f(x)=\sum_{k=0}^{\infty}(-1)^{k} \frac{\Gamma(1+\alpha)}{\Gamma(1+k) \Gamma(\alpha-k+1)} f[x+(\alpha-k) h],
$$

where $f: R \rightarrow R, x \rightarrow f(x)$ denote a continuous (but not necessarily differentiable) function and $h$ denotes a constant discretization span. An alternative, which is strictly equivalent to (2), is the following expression as

$$
\begin{array}{r}
f^{\alpha}(x)=\frac{1}{\Gamma(1-\alpha)} \frac{d}{d x} \int_{0}^{x}(x-\xi)^{-\alpha}[f(\xi)-f(0)] d \xi, \\
0<\alpha<1, \\
f^{\alpha}(x)=\left(f^{(n)}(x)\right)^{(\alpha-n)}, \quad n \leq \alpha \leq n+1, n \geq 1 .
\end{array}
$$

Some useful formulas of Jumarie's modified RiemannLiouville derivative were summarized in $[25,26]$; three of them are

$$
\begin{gathered}
D_{x}^{\alpha} x^{\gamma}=\frac{\Gamma(1+\gamma)}{\Gamma(1+\gamma-\alpha)} x^{\gamma-\alpha}, \quad \gamma>0, \\
D_{x}^{\alpha}(u(x) v(x))=v(x) D_{x}^{\alpha} u(x)+u(x) D_{x}^{\alpha} v(x), \\
D_{x}^{\alpha} f[u(x)]=f_{u}^{\prime}[u(x)] D_{x}^{\alpha} u(x)=D_{u}^{\alpha} f[u(x)]\left(u^{\prime}(x)\right)^{\alpha} .
\end{gathered}
$$

\section{Description of \\ the $\left(G^{\prime} / G\right)$-Expansion Method}

In this section, we give the description of the $\left(G^{\prime} / G\right)$ expansion method $[14,15]$ for solving the nonlinear FDE as

$$
\begin{array}{r}
P\left(u, D_{t}^{\alpha} u, D_{x}^{\beta} u, D_{t}^{\alpha} D_{t}^{\alpha} u, D_{t}^{\alpha} D_{x}^{\beta} u, D_{x}^{\beta} D_{x}^{\beta} u \ldots\right)=0, \\
0<\alpha, \beta \leq 1,
\end{array}
$$

where $u$ is an unknown function and $P$ is a polynomial of $u$ and its partial fractional derivatives, in which the highest order derivatives and nonlinear terms are involved.

Step 1. Li and He [27] and He and Li [28] proposed a fractional complex transformation to convert fractional differential equations into ordinary differential equations (ODE), so all analytical methods devoted to the advanced calculus can be easily applied to the fractional calculus. The complex wave variable was as follows:

$$
u(x, t)=U(\xi), \quad \xi=\frac{R x^{\beta}}{\Gamma(\beta+1)}+\frac{S t^{\alpha}}{\Gamma(\alpha+1)},
$$

where $R$ and $S$ are nonzero arbitrary constants; the nonlinear FDE (6) is reduced to a nonlinear ODE:

$$
Q\left(U, U^{\prime}, U^{\prime \prime}, U^{\prime \prime \prime}, \ldots\right)=0
$$

where the prime denotes the derivation with respect to $\xi$.

Step 2. Suppose that the solution of ODE (8) can be expressed as a polynomial in $\left(G^{\prime} / G\right)$ as follows:

$$
U(\xi)=\sum_{i=0}^{n} a_{i}\left(\frac{G^{\prime}}{G}\right)^{i}
$$

where $a_{i}(i=0,1,2, \ldots, n)$ are constants to be determined later and $n$ is a positive integer that is given by the homogeneous balance principle, and $G=G(\xi)$ satisfies the second order linear differential equation:

$$
G^{\prime \prime}+\lambda G^{\prime}+\mu G=0,
$$


where $\lambda$ and $\mu$ are real constants. Using the general solutions of (10), we have

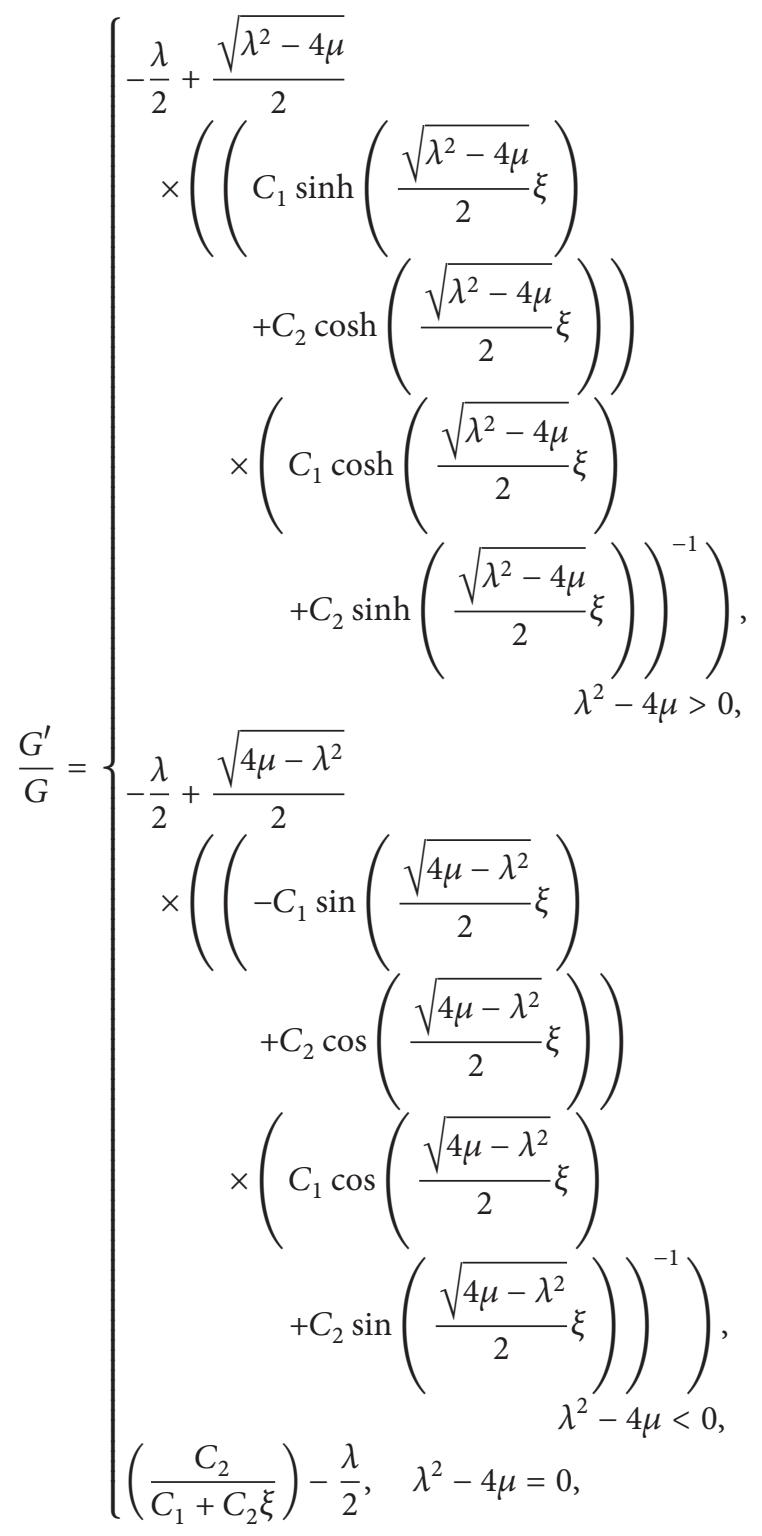

where $C_{1}$ and $C_{2}$ are arbitrary constants.

Step 3. Substituting (9) along with (10) into (8), collecting all terms with the same order of $\left(G^{\prime} / G\right)$ together, the left-hand side of $(8)$ is converted into a polynomial in $\left(G^{\prime} / G\right)$, and then setting coefficients of the polynomial to zero yields a set of algebraic equations for $R, S, \lambda$, and $\mu$ and $a_{i}(i=0,1, \ldots, n)$.

Step 4. Solving the algebraic equations obtained in Step 3, the constants $R, S, \lambda$, and $\mu$ and $a_{i}(i=0,1, \ldots, n)$ can be expressed. Substituting these values into expression (9), we can obtain the general form of the exact solution of (8).

Step 5. Substituting the exact solutions of (10) into the general form of exact solution obtained in Step 4, then we can obtain the exact solutions of (6).

\section{Exact Solutions of (1)}

In this section, we use above the $\left(G^{\prime} / G\right)$-expansion method to explore the exact solutions of (1).

Let

$$
\begin{aligned}
u(x, t) & =U(\xi), \quad v(x, t)=V(\xi), \\
\xi & =\frac{R x^{\alpha}}{\Gamma(\alpha+1)}+\frac{S t^{\alpha}}{\Gamma(\alpha+1)},
\end{aligned}
$$

where $R$ and $S$ are nonzero constants, and substituting (12) into (1), we obtain

$$
\begin{aligned}
& S V^{\prime}+R U^{\prime}+R U V^{\prime}+R V U^{\prime}+a R^{3} U^{\prime \prime \prime}-b R^{2} S V^{\prime \prime \prime}=0, \\
& S U^{\prime}+R V^{\prime}+R U U^{\prime}+c R^{3} V^{\prime \prime \prime}-d R^{2} S U^{\prime \prime \prime}=0 .
\end{aligned}
$$

We suppose that (13) has the solution in the form

$$
\begin{aligned}
& U(\xi)=\sum_{i=0}^{n} a_{i}\left(\frac{G^{\prime}}{G}\right)^{i}, \\
& V(\xi)=\sum_{j=0}^{m} b_{j}\left(\frac{G^{\prime}}{G}\right)^{j} .
\end{aligned}
$$

Balancing the highest order derivative terms and nonlinear terms in (13), we get $m=n=2$. So we can write

$$
\begin{aligned}
& U(\xi)=a_{0}+a_{1}\left(\frac{G^{\prime}}{G}\right)+a_{2}\left(\frac{G^{\prime}}{G}\right)^{2}, \\
& V(\xi)=b_{0}+b_{1}\left(\frac{G^{\prime}}{G}\right)+b_{2}\left(\frac{G^{\prime}}{G}\right)^{2},
\end{aligned}
$$

where $a_{0}, a_{1}, a_{2}, b_{0}, b_{1}$, and $b_{2}$ are constants to be determined later.

Substituting (15) along with (10) into (13), collecting all the terms of powers of $\left(G^{\prime} / G\right)$, the left-hand side of $(13)$ is converted into a polynomial in $\left(G^{\prime} / G\right)$, and then setting each coefficient to zero yields a set of algebraic equations for $R, S$, $\lambda, \mu, a_{0}, a_{1}, a_{2}, b_{0}, b_{1}, b_{2}$. Solving the set of algebraic equations by Maple, we get the following results.

Case 1. Consider

$$
\begin{aligned}
& a_{0}=\frac{8 a_{2}^{2} \mu+a_{2}^{2} \lambda^{2}-12}{12 a_{2}}+\frac{2 c-a a_{2}^{2}}{a_{2}(b-2 d)}, \quad a_{1}=a_{2} \lambda, \\
& b_{0}=\frac{8 a_{2}^{2} \mu-12 a_{2}^{2}+a_{2}^{2} \lambda^{2}+12}{12 a_{2}^{2}}, \quad b_{1}=\lambda, \quad b_{2}=1,
\end{aligned}
$$




$$
\begin{aligned}
& R=\frac{a_{2}}{2} \sqrt{\frac{b-2 d}{3 d a a_{2}^{2}-3 c b}}, \\
& S=\frac{a a_{2}^{2}-2 c}{6\left(d a a_{2}^{2}-c b\right) \sqrt{(b-2 d) /\left(3 d a a_{2}^{2}-3 c b\right)}},
\end{aligned}
$$

where $a_{2}$ is nonzero arbitrary constant.

Substituting (16) into (15), we obtain the following formal solution of (13):

$$
\begin{aligned}
U(\xi)= & \frac{8 a_{2}^{2} \mu+a_{2}^{2} \lambda^{2}-12}{12 a_{2}}+\frac{2 c-a a_{2}^{2}}{a_{2}(b-2 d)} \\
& +a_{2} \lambda\left(\frac{G^{\prime}}{G}\right)+a_{2}\left(\frac{G^{\prime}}{G}\right)^{2}, \\
V(\xi)= & \frac{8 a_{2}^{2} \mu-12 a_{2}^{2}+a_{2}^{2} \lambda^{2}+12}{12 a_{2}^{2}}+\lambda\left(\frac{G^{\prime}}{G}\right)+\left(\frac{G^{\prime}}{G}\right)^{2},
\end{aligned}
$$

where $\xi=\left(a_{2} / 2\right) \sqrt{(b-2 d) /\left(3 d a a_{2}^{2}-3 c b\right)}\left(x^{\alpha} / \Gamma(\alpha+1)\right)+$ $\left(\left(a a_{2}^{2}-2 c\right) / 6\left(d a a_{2}^{2}-c b\right) \sqrt{(b-2 d) /\left(3 d a a_{2}^{2}-3 c b\right)}\right)\left(t^{\alpha} / \Gamma(\alpha+\right.$ 1)).

Substituting the general solution of (10) into (17), we obtain the three types of traveling wave solutions depending on the sign of $\Delta=\lambda^{2}-4 \mu$.

If $\Delta=\lambda^{2}-4 \mu>0$, we have the following general hyperbolic traveling wave solutions of (1):

$u_{1}(x, t)$

$$
\begin{aligned}
& =\frac{8 a_{2}^{2} \mu+a_{2}^{2} \lambda^{2}-12}{12 a_{2}}+\frac{2 c-a a_{2}^{2}}{a_{2}(b-2 d)}+a_{2} \lambda \\
& \times\left[-\frac{\lambda}{2}+\frac{\sqrt{\Delta}}{2}\right. \\
& \left.\quad \times\left(\frac{C_{1} \sinh ((\sqrt{\Delta} / 2) \xi)+C_{2} \cosh ((\sqrt{\Delta} / 2) \xi)}{C_{1} \cosh ((\sqrt{\Delta} / 2) \xi)+C_{2} \sinh ((\sqrt{\Delta} / 2) \xi)}\right)\right]+a_{2} \\
& \times\left[-\frac{\lambda}{2}+\frac{\sqrt{\Delta}}{2}\right. \\
& \left.\quad \times\left(\frac{C_{1} \sinh ((\sqrt{\Delta} / 2) \xi)+C_{2} \cosh ((\sqrt{\Delta} / 2) \xi)}{C_{1} \cosh ((\sqrt{\Delta} / 2) \xi)+C_{2} \sinh ((\sqrt{\Delta} / 2) \xi)}\right)\right]^{2},
\end{aligned}
$$

$v_{1}(x, t)$

$=\frac{8 a_{2}^{2} \mu-12 a_{2}^{2}+a_{2}^{2} \lambda^{2}+12}{12 a_{2}^{2}}+\lambda$

$$
\begin{aligned}
\times[- & \frac{\lambda}{2}+\frac{\sqrt{\Delta}}{2} \\
& \left.\times\left(\frac{C_{1} \sinh ((\sqrt{\Delta} / 2) \xi)+C_{2} \cosh ((\sqrt{\Delta} / 2) \xi)}{C_{1} \cosh ((\sqrt{\Delta} / 2) \xi)+C_{2} \sinh ((\sqrt{\Delta} / 2) \xi)}\right)\right] \\
+ & {\left[-\frac{\lambda}{2}+\frac{\sqrt{\Delta}}{2}\right.} \\
& \left.\times\left(\frac{C_{1} \sinh ((\sqrt{\Delta} / 2) \xi)+C_{2} \cosh ((\sqrt{\Delta} / 2) \xi)}{C_{1} \cosh ((\sqrt{\Delta} / 2) \xi)+C_{2} \sinh ((\sqrt{\Delta} / 2) \xi)}\right)\right]^{2},
\end{aligned}
$$

where $\xi=\left(a_{2} / 2\right) \sqrt{(b-2 d) /\left(3 d a a_{2}^{2}-3 c b\right)}\left(x^{\alpha} / \Gamma(\alpha+1)\right)+$ $\left(\left(a a_{2}^{2}-2 c\right) / 6\left(d a a_{2}^{2}-c b\right) \sqrt{(b-2 d) /\left(3 d a a_{2}^{2}-3 c b\right)}\right)\left(t^{\alpha} / \Gamma(\alpha+\right.$ 1)).

In particular, setting $C_{2}=0$ and $C_{1} \neq 0$, then (18) can be written as

$$
\begin{aligned}
u_{1(1)} & (x, t) \\
= & \frac{8 a_{2}^{2} \mu+a_{2}^{2} \lambda^{2}-12}{12 a_{2}}+\frac{2 c-a a_{2}^{2}}{a_{2}(b-2 d)}+a_{2} \lambda \\
& \times\left[-\frac{\lambda}{2}+\frac{\sqrt{\Delta}}{2} \tanh \left(\frac{\sqrt{\Delta}}{2} \xi\right)\right] \\
& +a_{2}\left[-\frac{\lambda}{2}+\frac{\sqrt{\Delta}}{2} \tanh \left(\frac{\sqrt{\Delta}}{2} \xi\right)\right]^{2}, \\
v_{1(1)} & (x, t) \\
= & \frac{8 a_{2}^{2} \mu-12 a_{2}^{2}+a_{2}^{2} \lambda^{2}+12}{12 a_{2}^{2}}+\lambda \\
& \times\left[-\frac{\lambda}{2}+\frac{\sqrt{\Delta}}{2} \tanh \left(\frac{\sqrt{\Delta}}{2} \xi\right)\right] \\
& +\left[-\frac{\lambda}{2}+\frac{\sqrt{\Delta}}{2} \tanh \left(\frac{\sqrt{\Delta}}{2} \xi\right)\right]^{2},
\end{aligned}
$$

where $\xi=\left(a_{2} / 2\right) \sqrt{(b-2 d) /\left(3 d a a_{2}^{2}-3 c b\right)}\left(x^{\alpha} / \Gamma(\alpha+1)\right)+$ $\left(\left(a a_{2}^{2}-2 c\right) / 6\left(d a a_{2}^{2}-c b\right) \sqrt{(b-2 d) /\left(3 d a a_{2}^{2}-3 c b\right)}\right)\left(t^{\alpha} / \Gamma(\alpha+\right.$ 1)).

In particular, setting $C_{1}=0$ and $C_{2} \neq 0$, then (18) can be written as

$$
\begin{aligned}
& u_{1(2)}(x, t) \\
& =\frac{8 a_{2}^{2} \mu+a_{2}^{2} \lambda^{2}-12}{12 a_{2}}+\frac{2 c-a a_{2}^{2}}{a_{2}(b-2 d)}+a_{2} \lambda
\end{aligned}
$$




$$
\begin{gathered}
\times\left[-\frac{\lambda}{2}+\frac{\sqrt{\Delta}}{2} \operatorname{coth}\left(\frac{\sqrt{\Delta}}{2} \xi\right)\right] \\
+a_{2}\left[-\frac{\lambda}{2}+\frac{\sqrt{\Delta}}{2} \operatorname{coth}\left(\frac{\sqrt{\Delta}}{2} \xi\right)\right]^{2}, \\
v_{1(2)}(x, t) \\
=\frac{8 a_{2}^{2} \mu-12 a_{2}^{2}+a_{2}^{2} \lambda^{2}+12}{12 a_{2}^{2}}+\lambda \\
\times\left[-\frac{\lambda}{2}+\frac{\sqrt{\Delta}}{2} \operatorname{coth}\left(\frac{\sqrt{\Delta}}{2} \xi\right)\right] \\
+\left[-\frac{\lambda}{2}+\frac{\sqrt{\Delta}}{2} \operatorname{coth}\left(\frac{\sqrt{\Delta}}{2} \xi\right)\right]^{2},
\end{gathered}
$$

where $\xi=\left(a_{2} / 2\right) \sqrt{(b-2 d) /\left(3 d a a_{2}^{2}-3 c b\right)}\left(x^{\alpha} / \Gamma(\alpha+1)\right)+$ $\left(\left(a a_{2}^{2}-2 c\right) / 6\left(d a a_{2}^{2}-c b\right) \sqrt{(b-2 d) /\left(3 d a a_{2}^{2}-3 c b\right)}\right)\left(t^{\alpha} / \Gamma(\alpha+\right.$ 1)).

If $\Delta=\lambda^{2}-4 \mu<0$, we have the following general trigonometric function solutions of (1):

$$
\begin{aligned}
& u_{2}(x, t) \\
& =\frac{8 a_{2}^{2} \mu+a_{2}^{2} \lambda^{2}-12}{12 a_{2}}+\frac{2 c-a a_{2}^{2}}{a_{2}(b-2 d)}+a_{2} \lambda \\
& \times\left[-\frac{\lambda}{2}+\frac{\sqrt{-\Delta}}{2}\right. \\
& \left.\times\left(\frac{-C_{1} \sin ((\sqrt{-\Delta} / 2) \xi)+C_{2} \cos ((\sqrt{-\Delta} / 2) \xi)}{C_{1} \cos ((\sqrt{-\Delta} / 2) \xi)+C_{2} \sin ((\sqrt{-\Delta} / 2) \xi)}\right)\right] \\
& +a_{2}\left[-\frac{\lambda}{2}+\frac{\sqrt{-\Delta}}{2}\right. \\
& \left.\times\left(\frac{-C_{1} \sin ((\sqrt{-\Delta} / 2) \xi)+C_{2} \cos ((\sqrt{-\Delta} / 2) \xi)}{C_{1} \cos ((\sqrt{-\Delta} / 2) \xi)+C_{2} \sin ((\sqrt{-\Delta} / 2) \xi)}\right)\right]^{2},
\end{aligned}
$$

$v_{2}(x, t)$

$$
\begin{aligned}
= & \frac{8 a_{2}^{2} \mu-12 a_{2}^{2}+a_{2}^{2} \lambda^{2}+12}{12 a_{2}^{2}}+\lambda \\
\times & {\left[-\frac{\lambda}{2}+\frac{\sqrt{-\Delta}}{2}\right.} \\
& \left.\times\left(\frac{-C_{1} \sin ((\sqrt{-\Delta} / 2) \xi)+C_{2} \cos ((\sqrt{-\Delta} / 2) \xi)}{C_{1} \cos ((\sqrt{-\Delta} / 2) \xi)+C_{2} \sin ((\sqrt{-\Delta} / 2) \xi)}\right)\right]
\end{aligned}
$$

$$
\begin{aligned}
& +\left[-\frac{\lambda}{2}+\frac{\sqrt{-\Delta}}{2}\right. \\
& \left.\quad \times\left(\frac{-C_{1} \sin ((\sqrt{-\Delta} / 2) \xi)+C_{2} \cos ((\sqrt{-\Delta} / 2) \xi)}{C_{1} \cos ((\sqrt{-\Delta} / 2) \xi)+C_{2} \sin ((\sqrt{-\Delta} / 2) \xi)}\right)\right]^{2},
\end{aligned}
$$

where $\xi=\left(a_{2} / 2\right) \sqrt{(b-2 d) /\left(3 d a a_{2}^{2}-3 c b\right)}\left(x^{\alpha} / \Gamma(\alpha+1)\right)+$ $\left(\left(a a_{2}^{2}-2 c\right) / 6\left(d a a_{2}^{2}-c b\right) \sqrt{(b-2 d) /\left(3 d a a_{2}^{2}-3 c b\right)}\right)\left(t^{\alpha} / \Gamma(\alpha+\right.$ 1)).

In particular, setting $C_{2}=0$ and $C_{1} \neq 0$, then (21) can be written as

$$
\begin{aligned}
u_{2(1)} & (x, t) \\
= & \frac{8 a_{2}^{2} \mu+a_{2}^{2} \lambda^{2}-12}{12 a_{2}}+\frac{2 c-a a_{2}^{2}}{a_{2}(b-2 d)}+a_{2} \lambda \\
& \times\left[-\frac{\lambda}{2}-\frac{\sqrt{-\Delta}}{2} \tan \left(\frac{\sqrt{-\Delta}}{2} \xi\right)\right] \\
& +a_{2}\left[-\frac{\lambda}{2}-\frac{\sqrt{-\Delta}}{2} \tan \left(\frac{\sqrt{-\Delta}}{2} \xi\right)\right]^{2}, \\
v_{2(1)} & (x, t) \\
= & \frac{8 a_{2}^{2} \mu-12 a_{2}^{2}+a_{2}^{2} \lambda^{2}+12}{12 a_{2}^{2}}+\lambda \\
& \times\left[-\frac{\lambda}{2}-\frac{\sqrt{-\Delta}}{2} \tan \left(\frac{\sqrt{-\Delta}}{2} \xi\right)\right] \\
& +\left[-\frac{\lambda}{2}-\frac{\sqrt{-\Delta}}{2} \tan \left(\frac{\sqrt{-\Delta}}{2} \xi\right)\right]^{2},
\end{aligned}
$$

where $\xi=\left(a_{2} / 2\right) \sqrt{(b-2 d) /\left(3 d a a_{2}^{2}-3 c b\right)}\left(x^{\alpha} / \Gamma(\alpha+1)\right)+$ $\left(\left(a a_{2}^{2}-2 c\right) / 6\left(d a a_{2}^{2}-c b\right) \sqrt{(b-2 d) /\left(3 d a a_{2}^{2}-3 c b\right)}\right)\left(t^{\alpha} / \Gamma(\alpha+\right.$ 1)).

In particular, setting $C_{1}=0$ and $C_{2} \neq 0$, then (21) can be written as

$$
\begin{aligned}
u_{2(2)}( & x, t) \\
= & \frac{8 a_{2}^{2} \mu+a_{2}^{2} \lambda^{2}-12}{12 a_{2}}+\frac{2 c-a a_{2}^{2}}{a_{2}(b-2 d)}+a_{2} \lambda \\
& \times\left[-\frac{\lambda}{2}+\frac{\sqrt{-\Delta}}{2} \cot \left(\frac{\sqrt{-\Delta}}{2} \xi\right)\right] \\
& +a_{2}\left[-\frac{\lambda}{2}+\frac{\sqrt{-\Delta}}{2} \cot \left(\frac{\sqrt{-\Delta}}{2} \xi\right)\right]^{2},
\end{aligned}
$$




$$
\begin{aligned}
v_{2(2)}(x, t) & \\
= & \frac{8 a_{2}^{2} \mu-12 a_{2}^{2}+a_{2}^{2} \lambda^{2}+12}{12 a_{2}^{2}}+\lambda \\
& \times\left[-\frac{\lambda}{2}+\frac{\sqrt{-\Delta}}{2} \cot \left(\frac{\sqrt{-\Delta}}{2} \xi\right)\right] \\
+ & {\left[-\frac{\lambda}{2}+\frac{\sqrt{-\Delta}}{2} \cot \left(\frac{\sqrt{-\Delta}}{2} \xi\right)\right]^{2}, }
\end{aligned}
$$

where $\xi=\left(a_{2} / 2\right) \sqrt{(b-2 d) /\left(3 d a a_{2}^{2}-3 c b\right)}\left(x^{\alpha} / \Gamma(\alpha+1)\right)+$ $\left(\left(a a_{2}^{2}-2 c\right) / 6\left(d a a_{2}^{2}-c b\right) \sqrt{(b-2 d) /\left(3 d a a_{2}^{2}-3 c b\right)}\right)\left(t^{\alpha} / \Gamma(\alpha+\right.$ 1)).

If $\Delta=\lambda^{2}-4 \mu=0$, we have the following general rational function solutions of (1):

$$
\begin{aligned}
& u_{3}(x, t) \\
& =\frac{8 a_{2}^{2} \mu+a_{2}^{2} \lambda^{2}-12}{12 a_{2}}+\frac{2 c-a a_{2}^{2}}{a_{2}(b-2 d)}+a_{2} \lambda \\
& \quad \times\left[\left(\frac{C_{2}}{C_{1}+C_{2} \xi}\right)-\frac{\lambda}{2}\right]+a_{2}\left[\left(\frac{C_{2}}{C_{1}+C_{2} \xi}\right)-\frac{\lambda}{2}\right]^{2}, \\
& v_{3}(x, t) \\
& =\frac{8 a_{2}^{2} \mu-12 a_{2}^{2}+a_{2}^{2} \lambda^{2}+12}{12 a_{2}^{2}}+\lambda \\
& \quad \times\left[\left(\frac{C_{2}}{C_{1}+C_{2} \xi}\right)-\frac{\lambda}{2}\right]+\left[\left(\frac{C_{2}}{C_{1}+C_{2} \xi}\right)-\frac{\lambda}{2}\right]^{2},
\end{aligned}
$$

where $\xi=\left(a_{2} / 2\right) \sqrt{(b-2 d) /\left(3 d a a_{2}^{2}-3 c b\right)}\left(x^{\alpha} / \Gamma(\alpha+1)\right)+$ $\left(\left(a a_{2}^{2}-2 c\right) / 6\left(d a a_{2}^{2}-c b\right) \sqrt{(b-2 d) /\left(3 d a a_{2}^{2}-3 c b\right)}\right)\left(t^{\alpha} / \Gamma(\alpha+\right.$ 1)).

Case 2. Consider

$$
\begin{aligned}
& a_{0}=\left(\frac{\lambda^{2}}{12}+\frac{2 \mu}{3}+\frac{a(b-8 c-6 d)}{2 c(3 b-2 d)}\right) \sqrt{\frac{4 d c-6 c b}{a b-6 d a}} \\
& a_{1}=\sqrt{\frac{4 d c-6 c b}{a b-6 d a}} \lambda, \quad a_{2}=\sqrt{\frac{4 d c-6 c b}{a b-6 d a}} \\
& b_{0}=\frac{\lambda^{2}}{12}+\frac{2 \mu}{3}-1+\frac{a(b-6 d)}{2 c(2 d-3 b)}, \quad b_{1}=\lambda \\
& b_{2}=1, \quad R=\sqrt{\frac{3 b-2 d}{6 a b+12 d a}}, \\
& S=\frac{2 \sqrt{(4 d c-6 c b) /(a b-6 d a)}}{3(b+2 d) \sqrt{(3 b-2 d) /(6 a b+12 d a)}} .
\end{aligned}
$$

Substituting (25) into (15), we obtain the following formal solution of (13):

$$
\begin{aligned}
U(\xi)= & \left(\frac{\lambda^{2}}{12}+\frac{2 \mu}{3}+\frac{a(b-8 c-6 d)}{2 c(3 b-2 d)}\right) \sqrt{\frac{4 d c-6 c b}{a b-6 d a}} \\
& +\sqrt{\frac{4 d c-6 c b}{a b-6 d a}} \lambda\left(\frac{G^{\prime}}{G}\right)+\sqrt{\frac{4 d c-6 c b}{a b-6 d a}}\left(\frac{G^{\prime}}{G}\right)^{2}, \\
V(\xi)= & \frac{\lambda^{2}}{12}+\frac{2 \mu}{3}-1+\frac{a(b-6 d)}{2 c(2 d-3 b)}+\lambda\left(\frac{G^{\prime}}{G}\right)+\left(\frac{G^{\prime}}{G}\right)^{2},
\end{aligned}
$$

where $\xi=\sqrt{(3 b-2 d) /(6 a b+12 d a)}\left(x^{\alpha} / \Gamma(\alpha+\right.$ 1)) $+(2 \sqrt{(4 d c-6 c b) /(a b-6 d a)} / 3(b \quad+$ $2 d) \sqrt{(3 b-2 d) /(6 a b+12 d a)})\left(t^{\alpha} / \Gamma(\alpha+1)\right)$.

Substituting the general solution of (10) into (26), we obtain the three types of traveling wave solutions depending on the sign of $\Delta=\lambda^{2}-4 \mu$.

If $\Delta=\lambda^{2}-4 \mu>0$, we have the following general hyperbolic traveling wave solutions of (1):

$$
\begin{aligned}
& u_{4}(x, t) \\
& =\left(\frac{\lambda^{2}}{12}+\frac{2 \mu}{3}+\frac{a(b-8 c-6 d)}{2 c(3 b-2 d)}\right) \\
& \times \sqrt{\frac{4 d c-6 c b}{a b-6 d a}}+\sqrt{\frac{4 d c-6 c b}{a b-6 d a}} \lambda \\
& \times\left[-\frac{\lambda}{2}+\frac{\sqrt{\Delta}}{2}\right. \\
& \left.\times\left(\frac{C_{1} \sinh ((\sqrt{\Delta} / 2) \xi)+C_{2} \cosh ((\sqrt{\Delta} / 2) \xi)}{C_{1} \cosh ((\sqrt{\Delta} / 2) \xi)+C_{2} \sinh ((\sqrt{\Delta} / 2) \xi)}\right)\right] \\
& +\sqrt{\frac{4 d c-6 c b}{a b-6 d a}} \\
& \times\left[-\frac{\lambda}{2}+\frac{\sqrt{\Delta}}{2}\right. \\
& \left.\times\left(\frac{C_{1} \sinh ((\sqrt{\Delta} / 2) \xi)+C_{2} \cosh ((\sqrt{\Delta} / 2) \xi)}{C_{1} \cosh ((\sqrt{\Delta} / 2) \xi)+C_{2} \sinh ((\sqrt{\Delta} / 2) \xi)}\right)\right]^{2} \\
& v_{4}(x, t) \\
& =\frac{\lambda^{2}}{12}+\frac{2 \mu}{3}-1+\frac{a(b-6 d)}{2 c(2 d-3 b)}+\lambda \\
& \times\left[-\frac{\lambda}{2}+\frac{\sqrt{\Delta}}{2}\right. \\
& \left.\times\left(\frac{C_{1} \sinh ((\sqrt{\Delta} / 2) \xi)+C_{2} \cosh ((\sqrt{\Delta} / 2) \xi)}{C_{1} \cosh ((\sqrt{\Delta} / 2) \xi)+C_{2} \sinh ((\sqrt{\Delta} / 2) \xi)}\right)\right]
\end{aligned}
$$




$$
\begin{aligned}
& +\left[-\frac{\lambda}{2}+\frac{\sqrt{\Delta}}{2}\right. \\
& \left.\quad \times\left(\frac{C_{1} \sinh ((\sqrt{\Delta} / 2) \xi)+C_{2} \cosh ((\sqrt{\Delta} / 2) \xi)}{C_{1} \cosh ((\sqrt{\Delta} / 2) \xi)+C_{2} \sinh ((\sqrt{\Delta} / 2) \xi)}\right)\right]^{2},
\end{aligned}
$$

where $\xi=\sqrt{(3 b-2 d) /(6 a b+12 d a)}\left(x^{\alpha} / \Gamma(\alpha+\right.$ 1)) $+(2 \sqrt{(4 d c-6 c b) /(a b-6 d a)} / 3(b+$ $2 d) \sqrt{(3 b-2 d) /(6 a b+12 d a)})\left(t^{\alpha} / \Gamma(\alpha+1)\right)$.

In particular, setting $C_{2}=0$ and $C_{1} \neq 0$, then (27) can be written as

$$
\begin{aligned}
u_{4(1)}(x, t) & \\
= & \left(\frac{\lambda^{2}}{12}+\frac{2 \mu}{3}+\frac{a(b-8 c-6 d)}{2 c(3 b-2 d)}\right) \sqrt{\frac{4 d c-6 c b}{a b-6 d a}} \\
& +\sqrt{\frac{4 d c-6 c b}{a b-6 d a}} \lambda \times\left[-\frac{\lambda}{2}+\frac{\sqrt{\Delta}}{2} \tanh \left(\frac{\sqrt{\Delta}}{2} \xi\right)\right] \\
& +\sqrt{\frac{4 d c-6 c b}{a b-6 d a}}\left[-\frac{\lambda}{2}+\frac{\sqrt{\Delta}}{2} \tanh \left(\frac{\sqrt{\Delta}}{2} \xi\right)\right]^{2}
\end{aligned}
$$

$$
v_{4(1)}(x, t)
$$

$$
\begin{gathered}
=\frac{\lambda^{2}}{12}+\frac{2 \mu}{3}-1+\frac{a(b-6 d)}{2 c(2 d-3 b)}+\lambda \\
\times\left[-\frac{\lambda}{2}+\frac{\sqrt{\Delta}}{2} \tanh \left(\frac{\sqrt{\Delta}}{2} \xi\right)\right] \\
+\left[-\frac{\lambda}{2}+\frac{\sqrt{\Delta}}{2} \tanh \left(\frac{\sqrt{\Delta}}{2} \xi\right)\right]^{2}
\end{gathered}
$$

where $\xi=\sqrt{(3 b-2 d) /(6 a b+12 d a)}\left(x^{\alpha} / \Gamma(\alpha+\right.$ 1)) $+(2 \sqrt{(4 d c-6 c b) /(a b-6 d a)} / 3(b+$ $2 d) \sqrt{(3 b-2 d) /(6 a b+12 d a)})\left(t^{\alpha} / \Gamma(\alpha+1)\right)$.

In particular, setting $C_{1}=0$ and $C_{2} \neq 0$, then (27) can be written as

$$
\begin{aligned}
u_{4(2)}(x, t) \\
=\left(\frac{\lambda^{2}}{12}+\frac{2 \mu}{3}+\frac{a(b-8 c-6 d)}{2 c(3 b-2 d)}\right) \sqrt{\frac{4 d c-6 c b}{a b-6 d a}} \\
+\sqrt{\frac{4 d c-6 c b}{a b-6 d a} \lambda \times\left[-\frac{\lambda}{2}+\frac{\sqrt{\Delta}}{2} \operatorname{coth}\left(\frac{\sqrt{\Delta}}{2} \xi\right)\right]} \\
+\sqrt{\frac{4 d c-6 c b}{a b-6 d a}}\left[-\frac{\lambda}{2}+\frac{\sqrt{\Delta}}{2} \operatorname{coth}\left(\frac{\sqrt{\Delta}}{2} \xi\right)\right]^{2},
\end{aligned}
$$

$$
\begin{aligned}
v_{4(2)}(x, t) & \\
= & \frac{\lambda^{2}}{12}+\frac{2 \mu}{3}-1+\frac{a(b-6 d)}{2 c(2 d-3 b)}+\lambda \\
\times & {\left[-\frac{\lambda}{2}+\frac{\sqrt{\Delta}}{2} \operatorname{coth}\left(\frac{\sqrt{\Delta}}{2} \xi\right)\right] } \\
+ & {\left[-\frac{\lambda}{2}+\frac{\sqrt{\Delta}}{2} \operatorname{coth}\left(\frac{\sqrt{\Delta}}{2} \xi\right)\right]^{2}, }
\end{aligned}
$$

where $\xi=\sqrt{(3 b-2 d) /(6 a b+12 d a)}\left(x^{\alpha} / \Gamma(\alpha+\right.$ 1)) $+\quad(2 \sqrt{(4 d c-6 c b) /(a b-6 d a)} / 3(b+$ $2 d) \sqrt{(3 b-2 d) /(6 a b+12 d a)})\left(t^{\alpha} / \Gamma(\alpha+1)\right)$.

If $\Delta=\lambda^{2}-4 \mu<0$, we have the following general trigonometric function solutions of (1):

$$
\begin{aligned}
& u_{5}(x, t) \\
& =\left(\frac{\lambda^{2}}{12}+\frac{2 \mu}{3}+\frac{a(b-8 c-6 d)}{2 c(3 b-2 d)}\right) \\
& \times \sqrt{\frac{4 d c-6 c b}{a b-6 d a}}+\sqrt{\frac{4 d c-6 c b}{a b-6 d a}} \lambda \\
& \times\left[-\frac{\lambda}{2}+\frac{\sqrt{-\Delta}}{2}\right. \\
& \left.\times\left(\frac{-C_{1} \sin ((\sqrt{-\Delta} / 2) \xi)+C_{2} \cos ((\sqrt{-\Delta} / 2) \xi)}{C_{1} \cos ((\sqrt{-\Delta} / 2) \xi)+C_{2} \sin ((\sqrt{-\Delta} / 2) \xi)}\right)\right] \\
& +\sqrt{\frac{4 d c-6 c b}{a b-6 d a}} \\
& \times\left[-\frac{\lambda}{2}+\frac{\sqrt{-\Delta}}{2}\right. \\
& \left.\times\left(\frac{-C_{1} \sin ((\sqrt{-\Delta} / 2) \xi)+C_{2} \cos ((\sqrt{-\Delta} / 2) \xi)}{C_{1} \cos ((\sqrt{-\Delta} / 2) \xi)+C_{2} \sin ((\sqrt{-\Delta} / 2) \xi)}\right)\right]^{2}, \\
& v_{5}(x, t) \\
& =\frac{\lambda^{2}}{12}+\frac{2 \mu}{3}-1+\frac{a(b-6 d)}{2 c(2 d-3 b)}+\lambda \\
& \times\left[-\frac{\lambda}{2}+\frac{\sqrt{-\Delta}}{2}\right. \\
& \left.\times\left(\frac{-C_{1} \sin ((\sqrt{-\Delta} / 2) \xi)+C_{2} \cos ((\sqrt{-\Delta} / 2) \xi)}{C_{1} \cos ((\sqrt{-\Delta} / 2) \xi)+C_{2} \sin ((\sqrt{-\Delta} / 2) \xi)}\right)\right]
\end{aligned}
$$




$$
\begin{aligned}
& +\left[-\frac{\lambda}{2}+\frac{\sqrt{-\Delta}}{2}\right. \\
& \left.\quad \times\left(\frac{-C_{1} \sin ((\sqrt{-\Delta} / 2) \xi)+C_{2} \cos ((\sqrt{-\Delta} / 2) \xi)}{C_{1} \cos ((\sqrt{-\Delta} / 2) \xi)+C_{2} \sin ((\sqrt{-\Delta} / 2) \xi)}\right)\right]^{2},
\end{aligned}
$$

where $\xi=\sqrt{(3 b-2 d) /(6 a b+12 d a)}\left(x^{\alpha} / \Gamma(\alpha+\right.$ 1)) $+(2 \sqrt{(4 d c-6 c b) /(a b-6 d a)} / 3(b+$ 2d) $\sqrt{(3 b-2 d) /(6 a b+12 d a)})\left(t^{\alpha} / \Gamma(\alpha+1)\right)$.

In particular, setting $C_{2}=0$ and $C_{1} \neq 0$, then (30) can be written as

$$
\begin{aligned}
u_{5(1)}( & x, t) \\
= & \left(\frac{\lambda^{2}}{12}+\frac{2 \mu}{3}+\frac{a(b-8 c-6 d)}{2 c(3 b-2 d)}\right) \\
& \times \sqrt{\frac{4 d c-6 c b}{a b-6 d a}}+\sqrt{\frac{4 d c-6 c b}{a b-6 d a} \lambda} \\
& \times\left[-\frac{\lambda}{2}-\frac{\sqrt{-\Delta}}{2} \tan \left(\frac{\sqrt{-\Delta}}{2} \xi\right)\right] \\
& +\sqrt{\frac{4 d c-6 c b}{a b-6 d a}}\left[-\frac{\lambda}{2}-\frac{\sqrt{-\Delta}}{2} \tan \left(\frac{\sqrt{-\Delta}}{2} \xi\right)\right]^{2},
\end{aligned}
$$

$v_{5(1)}(x, t)$

$$
\begin{gathered}
=\frac{\lambda^{2}}{12}+\frac{2 \mu}{3}-1+\frac{a(b-6 d)}{2 c(2 d-3 b)}+\lambda \\
\times\left[-\frac{\lambda}{2}-\frac{\sqrt{-\Delta}}{2} \tan \left(\frac{\sqrt{-\Delta}}{2} \xi\right)\right] \\
+\left[-\frac{\lambda}{2}-\frac{\sqrt{-\Delta}}{2} \tan \left(\frac{\sqrt{-\Delta}}{2} \xi\right)\right]^{2},
\end{gathered}
$$

where $\xi=\sqrt{(3 b-2 d) /(6 a b+12 d a)}\left(x^{\alpha} / \Gamma(\alpha+\right.$ 1)) $+(2 \sqrt{(4 d c-6 c b) /(a b-6 d a)} / 3(b+$ $2 d) \sqrt{(3 b-2 d) /(6 a b+12 d a)})\left(t^{\alpha} / \Gamma(\alpha+1)\right)$.

In particular, setting $C_{1}=0$ and $C_{2} \neq 0$, then (30) can be written as

$$
\begin{aligned}
\mathcal{u}_{5(2)}( & x, t) \\
= & \left(\frac{\lambda^{2}}{12}+\frac{2 \mu}{3}+\frac{a(b-8 c-6 d)}{2 c(3 b-2 d)}\right) \\
& \times \sqrt{\frac{4 d c-6 c b}{a b-6 d a}}+\sqrt{\frac{4 d c-6 c b}{a b-6 d a} \lambda} \\
& \times\left[-\frac{\lambda}{2}+\frac{\sqrt{-\Delta}}{2} \cot \left(\frac{\sqrt{-\Delta}}{2} \xi\right)\right] \\
& +\sqrt{\frac{4 d c-6 c b}{a b-6 d a}}\left[-\frac{\lambda}{2}+\frac{\sqrt{-\Delta}}{2} \cot \left(\frac{\sqrt{-\Delta}}{2} \xi\right)\right]^{2},
\end{aligned}
$$

$$
\begin{aligned}
v_{5(2)}( & x, t) \\
= & \frac{\lambda^{2}}{12}+\frac{2 \mu}{3}-1+\frac{a(b-6 d)}{2 c(2 d-3 b)}+\lambda \\
& \times\left[-\frac{\lambda}{2}+\frac{\sqrt{-\Delta}}{2} \cot \left(\frac{\sqrt{-\Delta}}{2} \xi\right)\right] \\
+ & {\left[-\frac{\lambda}{2}+\frac{\sqrt{-\Delta}}{2} \cot \left(\frac{\sqrt{-\Delta}}{2} \xi\right)\right]^{2}, }
\end{aligned}
$$

where $\xi=\sqrt{(3 b-2 d) /(6 a b+12 d a)}\left(x^{\alpha} / \Gamma(\alpha+\right.$ 1)) $+(2 \sqrt{(4 d c-6 c b) /(a b-6 d a)} / 3(b \quad+$ $2 d) \sqrt{(3 b-2 d) /(6 a b+12 d a)})\left(t^{\alpha} / \Gamma(\alpha+1)\right)$.

If $\Delta=\lambda^{2}-4 \mu=0$, we have the following general rational function solutions of (1):

$$
\begin{aligned}
u_{6}(x, t) & \left(\frac{\lambda^{2}}{12}+\frac{2 \mu}{3}+\frac{a(b-8 c-6 d)}{2 c(3 b-2 d)}\right) \\
& \times \sqrt{\frac{4 d c-6 c b}{a b-6 d a}+\sqrt{\frac{4 d c-6 c b}{a b-6 d a}} \lambda} \\
& \times\left[\left(\frac{C_{2}}{C_{1}+C_{2} \xi}\right)-\frac{\lambda}{2}\right] \\
& +\sqrt{\frac{4 d c-6 c b}{a b-6 d a}}\left[\left(\frac{C_{2}}{C_{1}+C_{2} \xi}\right)-\frac{\lambda}{2}\right]^{2}, \\
v_{6}(x, & t) \\
= & \frac{\lambda^{2}}{12}+\frac{2 \mu}{3}-1+\frac{a(b-6 d)}{2 c(2 d-3 b)}+\lambda \\
& \times\left[\left(\frac{C_{2}}{C_{1}+C_{2} \xi}\right)-\frac{\lambda}{2}\right]+\left[\left(\frac{C_{2}}{C_{1}+C_{2} \xi}\right)-\frac{\lambda}{2}\right]^{2},
\end{aligned}
$$

where $\xi=\sqrt{(3 b-2 d) /(6 a b+12 d a)}\left(x^{\alpha} / \Gamma(\alpha+\right.$ 1)) $+(2 \sqrt{(4 d c-6 c b) /(a b-6 d a)} / 3(b+$ $2 d) \sqrt{(3 b-2 d) /(6 a b+12 d a)})\left(t^{\alpha} / \Gamma(\alpha+1)\right)$.

\section{Conclusions}

The exact solutions of (1) are only reported in [21]. In this work, based on the fractional complex transformation and Jumarie's modified Riemann-Liouville derivative, we successfully obtained some new exact solutions of the space-time fractional bidirectional wave equations using the $\left(G^{\prime} / G\right)$ expansion method. These solutions are expressed by the hyperbolic functions, the trigonometric functions, and the rational functions. If we set the parameters in the obtained wider set of solutions as special values, a variety of special solutions like kink shaped, antikink shaped, and bell type solitary solutions are obtained. Though the exact solutions of (1) have been obtained via the fractional Riccati equation 
method [21], they are different from the solutions obtained in this paper. This method is very efficient and simple in finding the exact solutions for the nonlinear fractional differential equations.

\section{Conflict of Interests}

The authors declare that there is no conflict of interests regarding the publication of this paper.

\section{Acknowledgment}

This research was supported by the Natural Science Foundations of Yunnan Province, China (nos. 2011FZ193 and 2013FZ117).

\section{References}

[1] R. Hilfer, Applications of Fractional Calculus in Physics, World Scientific, River Edge, NJ, USA, 2000.

[2] A. A. Kilbas, H. M. Srivastava, and J. J. Trujillo, Theory and Applications of Fractional Differential Equations, Elsevier, Amsterdam, The Netherlands, 2006.

[3] X. Yang, "Local fractional integral transforms," Progress in Nonlinear Science, vol. 1, no. 4, pp. 1-225, 2011.

[4] R. Metzler and J. Klafter, "The random walk's guide to anomalous diffusion: a fractional dynamics approach," Physics Reports, vol. 339, no. 1, pp. 1-77, 2000.

[5] F. Santamaria, S. Wils, E. de Schutter, and G. J. Augustine, "Anomalous diffusion in Purkinje cell dendrites caused by spines," Neuron, vol. 52, no. 4, pp. 635-648, 2006.

[6] S. Guo and L. Mei, "The fractional variational iteration method using He's polynomials," Physics Letters A, vol. 375, no. 3, pp. 309-313, 2011.

[7] S. Zhang, Q. A. Zong, D. Liu, and Q. Gao, "A generalized expfunction method for fractional Riccati differential equations," Communications in Fractional Calculus, vol. 1, no. 1, pp. 48-51, 2010.

[8] A. Bekir, Ö. Güner, and A. C. Cevikel, "Fractional complex transform and exp-function methods for fractional differential equations," Abstract and Applied Analysis, vol. 2013, Article ID 426462, 8 pages, 2013.

[9] S. Zhang and H.-Q. Zhang, "Fractional sub-equation method and its applications to nonlinear fractional PDEs," Physics Letters A, vol. 375, no. 7, pp. 1069-1073, 2011.

[10] B. Tang, Y. He, L. Wei, and X. Zhang, "A generalized fractional sub-equation method for fractional differential equations with variable coefficients," Physics Letters A, vol. 376, no. 38-39, pp. 2588-2590, 2012.

[11] F. Meng, "A new approach for solving fractional partial differential equations," Journal of Applied Mathematics, vol. 2013, Article ID 256823, 5 pages, 2013.

[12] E. A.-B. Abdel-Salam and E. A. Yousif, "Solution of nonlinear space-time fractional differential equations using the fractional Riccati expansion method," Mathematical Problems in Engineering, vol. 2013, Article ID 846283, 6 pages, 2013.

[13] B. Lu, "The first integral method for some time fractional differential equations," Journal of Mathematical Analysis and Applications, vol. 395, no. 2, pp. 684-693, 2012.
[14] B. Zheng, " $\left(G^{\prime} / G\right)$-expansion method for solving fractional partial differential equations in the theory of mathematical physics," Communications in Theoretical Physics, vol. 58, no. 5, pp. 623-630, 2012.

[15] K. A. Gepreel and S. Omran, "Exact solutions for nonlinear partial fractional differential equations," Chinese Physics B, vol. 21, no. 11, Article ID 110204, 2012.

[16] M. Wang, X. Li, and J. Zhang, "The $\left(G^{\prime} / G\right)$-expansion method and travelling wave solutions of nonlinear evolution equations in mathematical physics," Physics Letters A, vol. 372, no. 4, pp. 417-423, 2008.

[17] A. Akgül, A. Kılıçman, and M. Inc, "Improved $\left(G^{\prime} / G\right)$ expansion method for the space and time fractional foam drainage and KdV equations," Abstract and Applied Analysis, vol. 2013, Article ID 414353, 7 pages, 2013.

[18] E. M. E. Zayed, Y. A. Amer, and R. M. A. Shohib, "Exact traveling wave solutions for nonlinear fractional partial differential equations using the improved $\left(G^{\prime} / G\right)$-expansion method," International Journal of Engineering and Applied Science, vol. 4, no. 7, pp. 18-31, 2014.

[19] K. A. Gepreel, T. A. Nofal, and F. M. Alotaibi, "Numerical solutions for the time and space fractional nonlinear partial differential equations," Journal of Applied Mathematics, vol. 2013, Article ID 482419, 12 pages, 2013.

[20] M. Younis and A. Zafar, "Travelling wave solutions of fractional order coupled Burgers' equations by $\left(G^{\prime} / G\right)$-expansion method," American Journal of Computational and Applied Mathematics, vol. 3, no. 2, pp. 81-85, 2013.

[21] B. Lu, "Bäcklund transformation of fractional Riccati equation and infinite sequence solutions of nonlinear fractional PDEs," Abstract and Applied Analysis, vol. 2014, Article ID 572052, 6 pages, 2014.

[22] J. L. Bona and M. Chen, "A Boussinesq system for twoway propagation of nonlinear dispersive waves," Physica D: Nonlinear Phenomena, vol. 116, no. 1-2, pp. 191-224, 1998.

[23] M. Chen, "Exact solutions of various Boussinesq systems," Applied Mathematics Letters, vol. 11, no. 5, pp. 45-49, 1998.

[24] J. Lee and R. Sakthivel, "New exact travelling wave solutions of bidirectional wave equations," Journal of Physics, vol. 76, no. 6, pp. 819-829, 2011.

[25] G. Jumarie, "Modified Riemann-Liouville derivative and fractional Taylor series of nondifferentiable functions further results," Computers \& Mathematics with Applications, vol. 51, no. 9-10, pp. 1367-1376, 2006.

[26] G. Jumarie, "Table of some basic fractional calculus formulae derived from a modified Riemann-Liouville derivative for nondifferentiable functions," Applied Mathematics Letters, vol. 22, no. 3, pp. 378-385, 2009.

[27] Z.-B. Li and J.-H. He, "Fractional complex transform for fractional differential equations," Mathematical \& Computational Applications, vol. 15, no. 5, pp. 970-973, 2010.

[28] J. He and Z. Li, "Converting fractional differential equations into partial differential equations," Thermal Science, vol. 16, no. 2, pp. 331-334, 2012. 


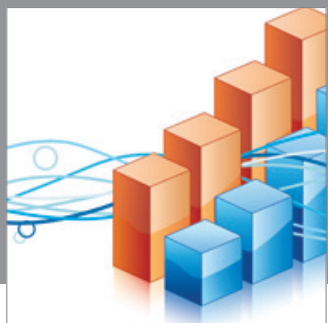

Advances in

Operations Research

mansans

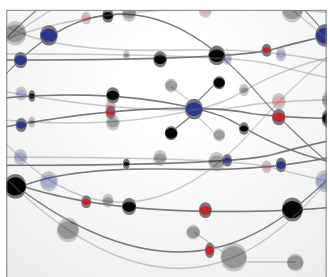

The Scientific World Journal
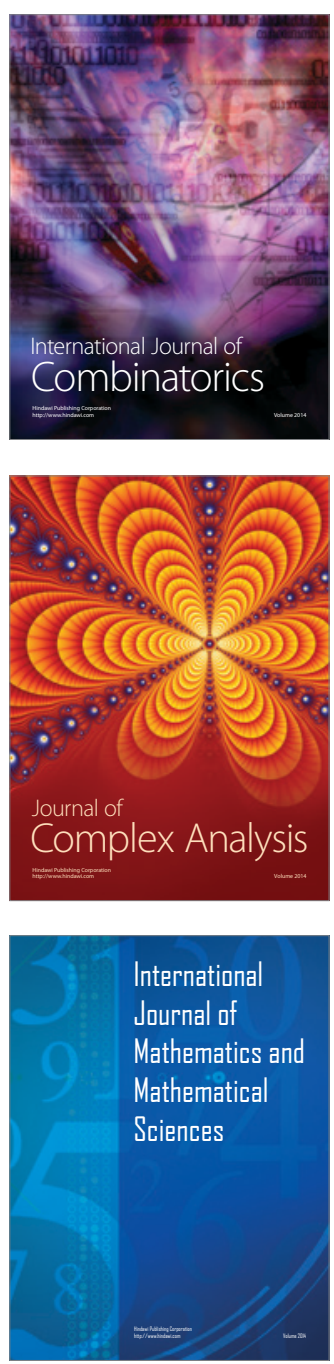
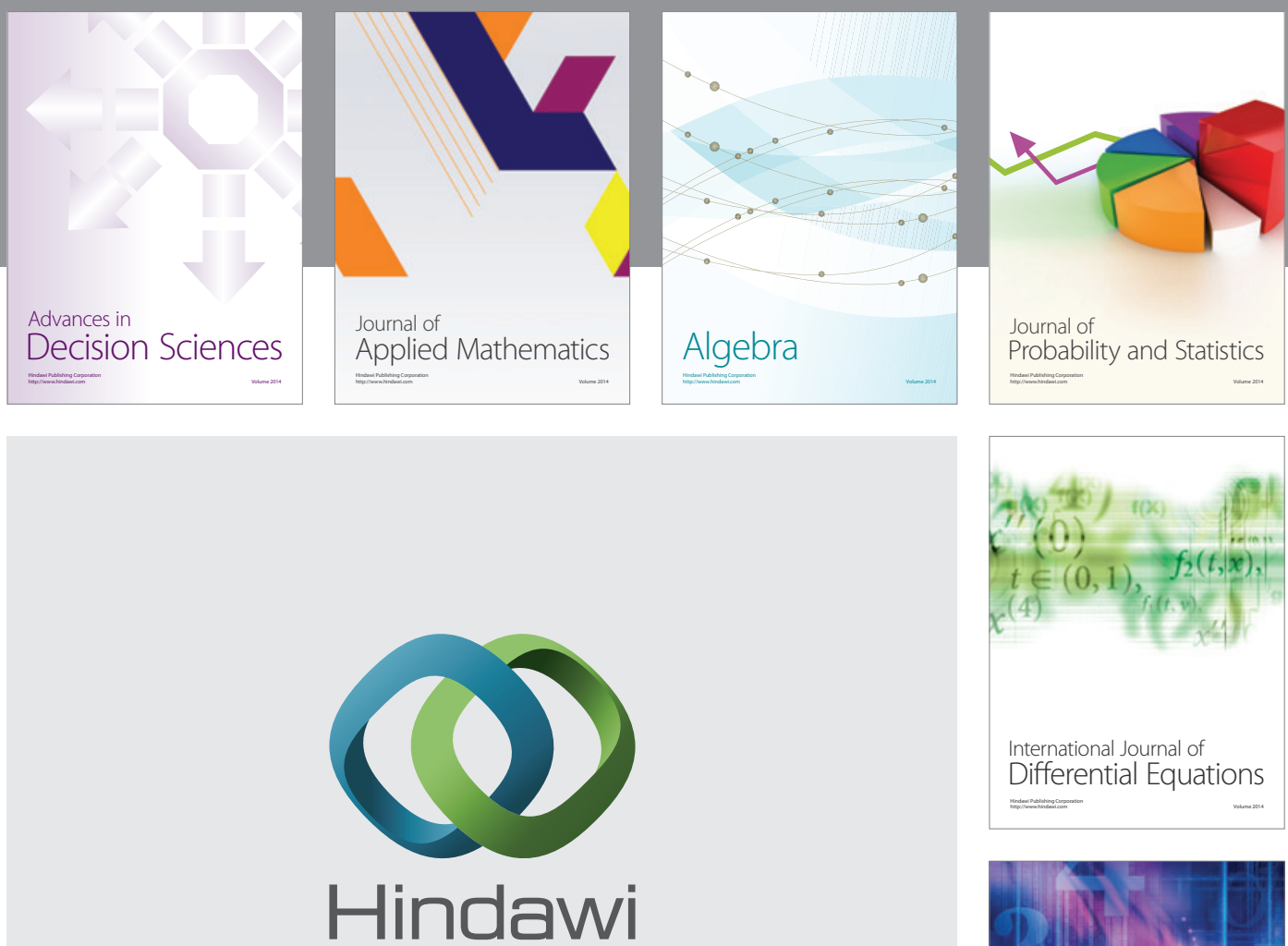

Submit your manuscripts at http://www.hindawi.com
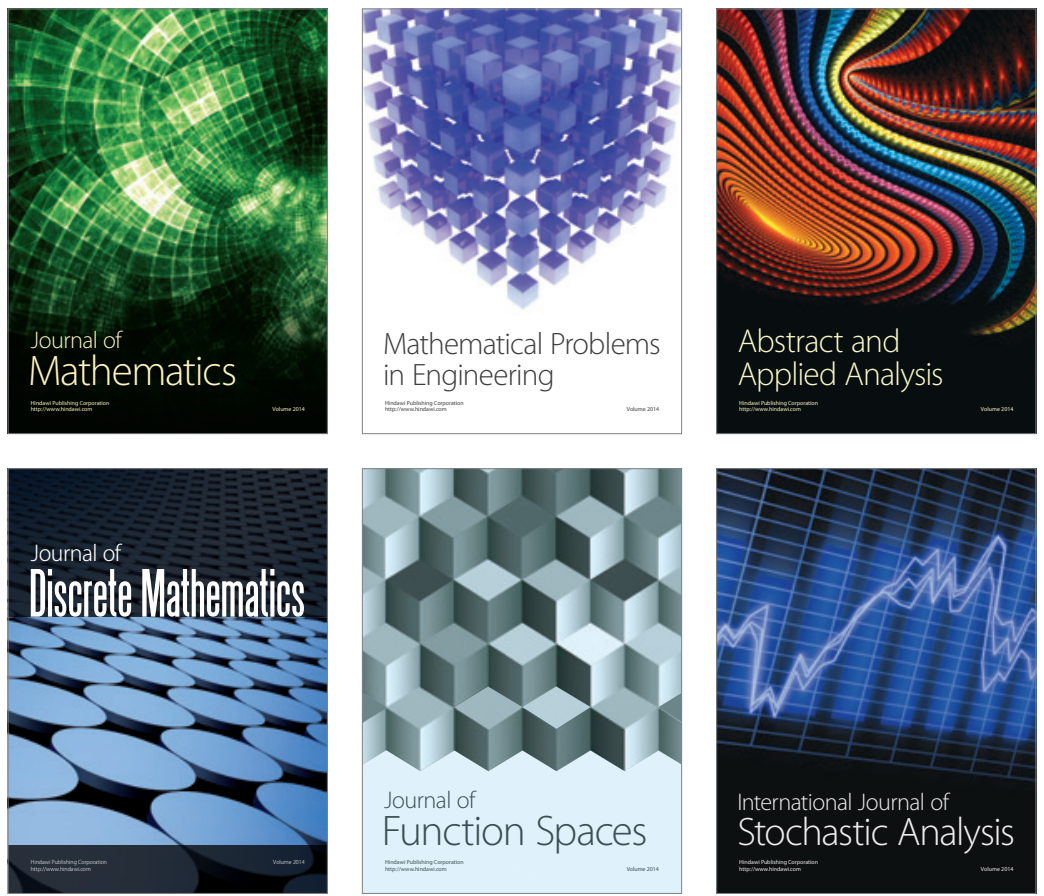

Journal of

Function Spaces

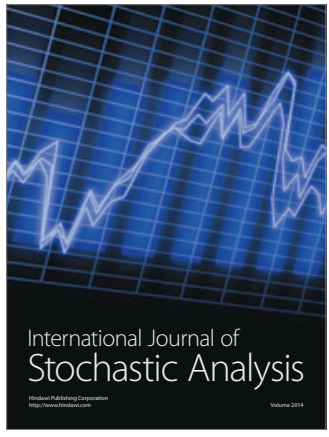

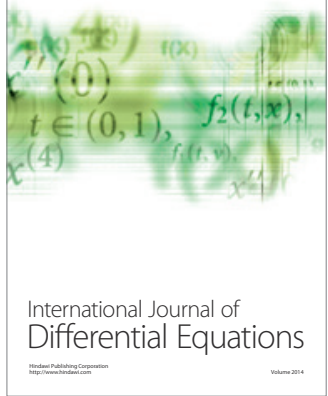
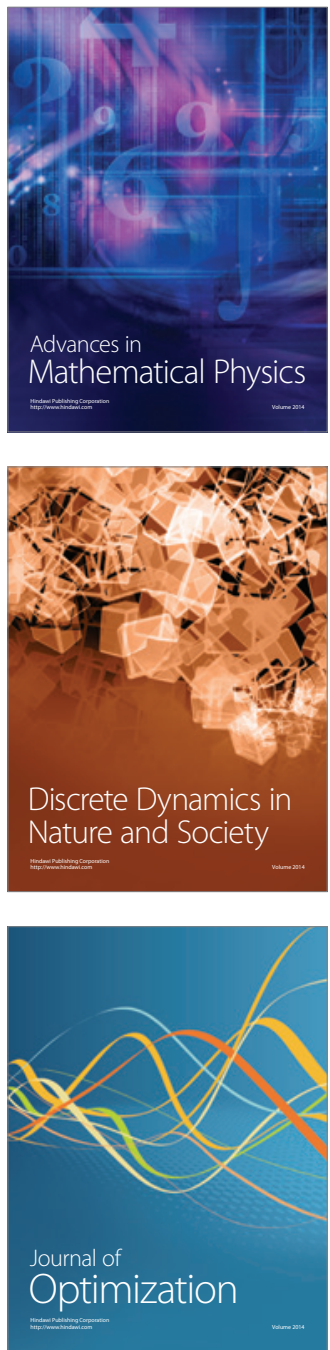